\title{
ON A CONVEXITY THEOREM OF RUSKAI AND WERNER AND RELATED RESULTS
}

\author{
HORST ALZER \\ Morsbacher Str. 10, 51545 Waldbröl, Germany \\ e-mail:alzerhorst@freenet.de
}

(Received 6 May, 2004; accepted 20 April, 2005)

Abstract. We show that the function

$$
V_{q}(x)=\frac{2 e^{x^{2}}}{\Gamma(q+1)} \int_{x}^{\infty} e^{-t^{2}}\left(t^{2}-x^{2}\right)^{q} d t \quad(-1<q \in \mathbf{R} ; 0<x \in \mathbf{R}),
$$

which has applications in the study of atoms in magnetic fields, satisfies certain monotonicity and convexity properties as well as inequalities. In particular, we prove that $1 / V_{q}$ is convex on $(0, \infty)$ if and only if $q \geq 0$. This extends a recent result of M. B. Ruskai and E. Werner, who established the convexity for all integers $q \geq 0$.

2000 Mathematics Subject Classification. 33E20, 26 D15.

1. Introduction. In an interesting paper published in 2000, M. B. Ruskai and E. Werner [8] discuss in detail the function

$$
V_{q}(x)=\frac{2 e^{x^{2}}}{\Gamma(q+1)} \int_{x}^{\infty} e^{-t^{2}}\left(t^{2}-x^{2}\right)^{q} d t \quad(-1<q \in \mathbf{R} ; 0<x \in \mathbf{R})
$$

and its extensions. $V_{q}(x)$ is also defined for $x=0$, if $q>-1 / 2$. The authors point out that their work was motivated by the fact that for an integer $q$ this function 'arises naturally' $\left[8\right.$, p. 436] in the study of atoms in magnetic fields. Indeed, $V_{q}$ can be regarded as one-dimensional regularization of the Coulomb potential. See [3], [4], and [8] for details and references.

The special case $q=0$ leads to Mills's ratio

$$
\frac{1}{\sqrt{2}} V_{0}\left(\frac{x}{\sqrt{2}}\right)=e^{x^{2} / 2} \int_{x}^{\infty} e^{-t^{2} / 2} d t
$$

which has applications in statistics. Inequalities for this and related functions are given in [7, Section 2.26].

A remarkable number theoretic property of

$$
\frac{V_{q}(0)}{\sqrt{\pi}}=2^{-2 q}\left(\begin{array}{c}
2 q \\
q
\end{array}\right)=\frac{1 \cdot 3 \cdot 5 \cdot \ldots \cdot(2 q-1)}{2 \cdot 4 \cdot 6 \cdot \ldots \cdot(2 q)} \quad(0 \leq q \in \mathbf{Z}),
$$

known as normalized binomial mid-coefficient, can be found in [2].

A central role in [8] is the study of convexity properties of $V_{q}(x)$. The authors show that the arithmetic mean of $V_{0}(x), \ldots, V_{n-1}(x)(n \geq 1)$ is convex on $(0, \infty)$ with respect to $x$. In particular, $x \mapsto V_{q}(x)$ is convex for $q=0$. But, this is not true, if $q>1 / 2$. In 
1993, M. Wirth [10] established that $1 / V_{0}$ is convex on $(0, \infty)$. Ruskai and Werner provide a substantial extension of this theorem. They prove that for all integers $q \geq 0$ the function $x \mapsto 1 / V_{q}(x)$ is convex on $(0, \infty)$. An application of this result reveals that $1 / V_{q}$ is subadditive, that is,

$$
\frac{1}{V_{q}(x+y)} \leq \frac{1}{V_{q}(x)}+\frac{1}{V_{q}(y)} \quad(x, y>0 ; 0 \leq q \in \mathbf{Z}) .
$$

The ratio $V_{q+1}(x) / V_{q}(x)(1 \leq q \in \mathbf{Z})$ is of importance in the proof of the convexity of $1 / V_{q}$. This ratio has an interesting monotonicity property: it is increasing with respect to $x$. The authors also study $V_{q}(x)$ as function of $q$. They establish that $q \mapsto V_{q}(x)$ and $q \mapsto-q V_{q}(x)(x>0)$ are decreasing.

It is our aim to continue the work of Ruskai and Werner. In Section 3, we determine all real parameters $p$ and $q$ such that $x \mapsto V_{p}(x)$ and $x \mapsto 1 / V_{q}(x)$ are convex on $(0, \infty)$. Moreover, we give an answer to the question: for which $q$ is $x \mapsto V_{q}(x)$ completely monotonic on $(0, \infty)$ ? And, we prove that for every $x>0$ the function $q \mapsto V_{q}(x)$ is convex on $(-1, \infty)$. In Section 4 , we extend and complement inequality (1.2). Further, we provide all parameters $q$ such that $x \mapsto V_{q}(x)$ is supermultiplicative on $(0, \infty)$, and we present a differential inequality involving $\left(V_{q}^{(k)}(x)\right)^{n}$ and $\left(V_{q}^{(n)}(x)\right)^{k}$. Finally, we study the monotonicity behaviour of the functions $x \mapsto V_{p}(x) / V_{q}(x)$ and $x \mapsto$ $V_{p}(x)-V_{q}(x)$.

2. Lemmas. In this section, we collect some lemmas, which we need to prove our theorems. First, we present integral representations for $V_{q}(x)$ and its first and second derivatives with respect to $x$.

LEMMA 1. For all $q>-1$ and $x>0$ we have

$$
\begin{gathered}
V_{q}(x)=\frac{1}{\Gamma(q+1)} \int_{0}^{\infty} e^{-\left(s x+s^{2} / 4\right)}\left(s x+s^{2} / 4\right)^{q} d s, \\
V_{q}(x)=\frac{x^{q+1 / 2}}{\Gamma(q+1)} \int_{0}^{\infty} e^{-x s} \frac{s^{q}}{(x+s)^{1 / 2}} d s \\
V_{q}^{\prime}(x)=-\frac{x^{q+1 / 2}}{\Gamma(q+1)} \int_{0}^{\infty} e^{-x s} \frac{s^{q}}{(x+s)^{3 / 2}} d s, \\
V_{q}^{\prime \prime}(x)=\frac{x^{q-1 / 2}}{\Gamma(q+1)} \int_{0}^{\infty} e^{-x s} \frac{(2 x-s) s^{q}}{(x+s)^{5 / 2}} d s .
\end{gathered}
$$

Proof. We substitute in (1.1) $t=x+s / 2$ and $t=\sqrt{x^{2}+x s}$, respectively, and obtain (2.1) and (2.2), respectively. Next, we set $s=u / x$ in (2.2). This yields

$$
V_{q}(x)=\frac{1}{\Gamma(q+1)} \int_{0}^{\infty} e^{-u} \frac{u^{q}}{\left(x^{2}+u\right)^{1 / 2}} d u .
$$

Further, if we differentiate (2.5) once and twice, respectively, and substitute $u=x s$, then we get (2.3) and (2.4), respectively.

Proofs for the next two lemmas are given in [8]. 
LemMA 2. Let $q>-1$ and $x>0$. The function $a \mapsto a V_{q}(a x)$ is strictly increasing on $(0, \infty)$.

LemMa 3. Let $q>-1$. Then we have the asymptotic formula

$$
V_{q}(x)=\frac{1}{x}-\frac{q+1}{2 x^{3}}+\frac{3(q+1)(q+2)}{8 x^{5}}+O\left(\frac{1}{x^{7}}\right) .
$$

The following integral inequality was first proved by P. L. Tchebyschef. References for this and related results can be found in [7, Section 2.5].

Lemma 4. Let $f, g:[a, b] \rightarrow \mathbf{R}$ be both increasing or both decreasing and let $p:$ $[a, b] \rightarrow[0, \infty)$ be integrable. Then

$$
\int_{a}^{b} p(x) f(x) d x \int_{a}^{b} p(x) g(x) d x \leq \int_{a}^{b} p(x) f(x) g(x) d x \int_{a}^{b} p(x) d x .
$$

Moreover, we need an inequality for convex functions due to M. Petrović [7, pp. 22-23].

Lemma 5. Let $f:[0, a] \rightarrow \mathbf{R}$ be convex. If $x_{j} \in[0, a](j=1, \ldots, n)$ and $x_{1}+\ldots+x_{n} \in[0, a]$, then

$$
f\left(x_{1}\right)+\ldots+f\left(x_{n}\right) \leq f\left(x_{1}+\ldots+x_{n}\right)+(n-1) f(0) .
$$

A function $f:(0, \infty) \rightarrow \mathbf{R}$ is called completely monotonic, if $f$ has derivatives of all orders and satisfies $(-1)^{n} f^{(n)}(x) \geq 0$ for all $x>0$ and $n=0,1,2, \ldots$ In particular, completely monotonic functions are decreasing and convex. These functions have numerous applications in probability theory, physics, and other branches. We refer to [1], where details and references can be found. The basic properties of completely monotonic functions are collected in [9, Chapter IV].

LEMMA 6. If $f$ is completely monotonic on $(0, \infty)$, then we have for all real numbers $x>0$ and integers $n, k$ with $n \geq k \geq 0$ :

$$
(-1)^{n k}\left(f^{(k)}(x)\right)^{n} \leq(-1)^{n k}\left(f^{(n)}(x)\right)^{k}(f(x))^{n-k} .
$$

A proof of Lemma 5 is given in [5].

3. Complete monotonicity and convexity. Our first theorem provides all parameters $q$ such that $V_{q}$ is completely monotonic on $(0, \infty)$.

THEOREM 1. Let $q>-1$ be a real number. The function $x \mapsto V_{q}(x)$ is completely monotonic on $(0, \infty)$ if and only if $q \in(-1,0]$.

Proof. Let $q \in(-1,0]$ and $x>0$ be real numbers. Further, let $n \geq 0$ be an integer. Using the Leibniz rule for the $n$-th derivative of a product we conclude from (2.1):

$$
(-1)^{n} V_{q}^{(n)}(x)=\frac{1}{\Gamma(q+1)} \int_{0}^{\infty} e^{-s(x+s / 4)} \sum_{\nu=0}^{n}\left(\begin{array}{l}
n \\
v
\end{array}\right) s^{q+n-v}\left(x+\frac{s}{4}\right)^{q-v} \prod_{j=0}^{\nu-1}(j-q) d s>0,
$$


which implies that $V_{q}$ is completely monotonic on $(0, \infty)$. Next, we show: if $q>0$, then $V_{q}$ is not convex and, thus not completely monotonic on $(0, \infty)$. We consider three cases.

Case 1: $0<q<1 / 2$.

Let

$$
\lambda_{q}(x, s)=\left(\frac{s}{x+s}\right)^{q} \text { and } \quad \mu_{q}(x, s)=\frac{s-2 x e^{x}}{(x+s)^{5 / 2-q}},
$$

where $s \in[0,1]$ and $x>0$ is sufficiently small. The function $s \mapsto \lambda_{q}(x, s)$ is increasing on $[0,1]$, so that we get

$$
\lambda_{q}(x, s) \mu_{q}(x, s) \geq \lambda_{q}\left(x, 2 x e^{x}\right) \mu_{q}(x, s) .
$$

Hence,

$$
\begin{aligned}
\int_{0}^{1} \lambda_{q}(x, s) \mu_{q}(x, s) d s \geq & \left(\frac{2 e^{x}}{1+2 e^{x}}\right)^{q} \int_{0}^{1} \frac{s-2 x e^{x}}{(x+s)^{5 / 2-q}} d s \\
= & \left(\frac{2 e^{x}}{1+2 e^{x}}\right)^{q} \frac{1}{(3 / 2-q)(1 / 2-q)}\left[x^{q-1 / 2}\left(1+e^{x}(2 q-1)\right)\right. \\
& \left.-(x+1)^{q-3 / 2}\left(x+x e^{x}(2 q-1)+3 / 2-q\right)\right] .
\end{aligned}
$$

Since $0<q<1 / 2$, we conclude that the expression on the right-hand side tends to $\infty$, if $x$ tends to 0 . This yields

$$
0<\int_{0}^{1} \frac{s^{q}}{(x+s)^{5 / 2}}\left(s-2 x e^{x}\right) d s .
$$

Using (3.1) we obtain

$$
\begin{aligned}
2 x \int_{0}^{1} e^{-x s} \frac{s^{q}}{(x+s)^{5 / 2}} d s & <2 x \int_{0}^{1} \frac{s^{q}}{(x+s)^{5 / 2}} d s<e^{-x} \int_{0}^{1} \frac{s^{q+1}}{(x+s)^{5 / 2}} d s \\
& <\int_{0}^{1} e^{-x s} \frac{s^{q+1}}{(x+s)^{5 / 2}} d s .
\end{aligned}
$$

Thus,

$$
\Gamma(q+1) x^{1 / 2-q} V_{q}^{\prime \prime}(x)<\int_{0}^{1} e^{-x s} \frac{(2 x-s) s^{q}}{(x+s)^{5 / 2}} d s<0
$$

for all sufficiently small $x$.

Case 2: $q=1 / 2$.

We define

$$
W\left(x^{2}\right)=\frac{\sqrt{\pi}}{2} V_{1 / 2}^{\prime \prime}(x)=\int_{0}^{\infty} e^{-t} t^{1 / 2} \frac{2 x^{2}-t}{\left(x^{2}+t\right)^{5 / 2}} d t .
$$

Let $y \in(0,1 / 2)$. We get

$$
\begin{aligned}
W(y) & \leq 2 y \int_{0}^{1} e^{-t} \frac{t^{1 / 2}}{(y+t)^{5 / 2}} d t-\int_{0}^{1} e^{-t} \frac{t^{3 / 2}}{(y+t)^{5 / 2}} d t \\
& \leq 2 y \int_{0}^{1} \frac{t^{1 / 2}}{(y+t)^{5 / 2}} d t-\frac{1}{e} \int_{0}^{1} \frac{t^{3 / 2}}{(y+t)^{5 / 2}} d t .
\end{aligned}
$$


Since

$$
\lim _{y \rightarrow 0} y \int_{0}^{1} \frac{t^{1 / 2}}{(y+t)^{5 / 2}} d t=\lim _{y \rightarrow 0} \frac{2}{3(y+1)^{3 / 2}}=\frac{2}{3} \quad \text { and } \lim _{y \rightarrow 0} \int_{0}^{1} \frac{t^{3 / 2}}{(y+t)^{5 / 2}} d t=\infty,
$$

we conclude that $W(y)$ is negative for sufficiently small $y$.

Case 3: $q>1 / 2$.

The substitution $s=t / x$ in (2.3) leads to

$$
V_{q}^{\prime}(x)=-\frac{x}{\Gamma(q+1)} \int_{0}^{\infty} e^{-t} \frac{t^{q}}{\left(x^{2}+t\right)^{3 / 2}} d t
$$

This implies $\lim _{x \rightarrow 0} V_{q}^{\prime}(x)=0$. Since $V_{q}^{\prime}(x)<0$ for $x>0$, we conclude that $V_{q}^{\prime}$ is not increasing on $(0, \infty)$.

REMARK 1. In particular, we have proved: the function $x \mapsto V_{q}(x)$ is convex on $(0, \infty)$ if and only if $q \in(-1,0]$.

REMARK 2. Since a completely monotonic function is log-convex, and a log-convex function is convex, we obtain: the function $x \mapsto V_{q}(x)$ is log-convex on $(0, \infty)$ if and only if $q \in(-1,0]$.

Next, we study the convexity of $1 / V_{q}$. Ruskai and Werner [8] conjecture that for all real numbers $q>-1$ the function $x \mapsto 1 / V_{q}(x)$ is convex on $(0, \infty)$. The following theorem reveals that this is true for all $q \geq 0$, but false for all $q \in(-1,0)$.

THEOREM 2. Let $q>-1$ be a real number. The function $x \mapsto 1 / V_{q}(x)$ is convex on $(0, \infty)$ if and only if $q \geq 0$. Moreover, if $q \geq 0$, then $1 / V_{q}$ is strictly convex on $(0, \infty)$.

Proof. Let $q \geq 0$ and $x>0$. Differentiation with respect to $x$ yields

$$
\left(V_{q}(x)\right)^{3}\left(\frac{1}{V_{q}(x)}\right)^{\prime \prime}=2\left(V_{q}^{\prime}(x)\right)^{2}-V_{q}(x) V_{q}^{\prime \prime}(x) .
$$

Using (2.2)-(2.4) and the convolution theorem we get

$$
\frac{(\Gamma(q+1))^{2}}{2 x^{2 q+1}}\left[2\left(V_{q}^{\prime}(x)\right)^{2}-V_{q}(x) V_{q}^{\prime \prime}(x)\right]=\int_{0}^{\infty} e^{-x t} \Lambda_{q}(x, t) d t
$$

where

$$
\Lambda_{q}(x, t)=\int_{0}^{t} \frac{[s(t-s)]^{q}}{[(x+s)(x+t-s)]^{5 / 2}}\left[(x+s)(x+t-s)-(1-s /(2 x))(x+t-s)^{2}\right] d s .
$$

Let $t>0$. We define

$$
\Theta_{q}(x, t)=8 x\left(\frac{2}{t}\right)^{2 q+2} \Lambda_{q}(x, t) .
$$

Next, we substitute $s=t(1+y) / 2$. This leads to

$$
\Theta_{q}(x, t)=\int_{-1}^{1} \Psi_{q}(x, t, y)\left[\alpha(t) y^{3}+\beta(x, t) y^{2}+\gamma(x, t) y+\delta(x, t)\right] d y,
$$

where

$$
\begin{gathered}
\Psi_{q}(x, t, y)=\left(1-y^{2}\right)^{q}\left[\left(x+\frac{t}{2}\right)^{2}-\left(\frac{t y}{2}\right)^{2}\right]^{-5 / 2}, \\
\alpha(t)=t^{2}, \quad \beta(x, t)=-12 x t-t^{2}, \quad \gamma(x, t)=20 x^{2}+8 x t-t^{2}, \quad \delta(x, t)=(2 x+t)^{2} .
\end{gathered}
$$


Since $y \mapsto \Psi_{q}(x, t, y)$ is even, we obtain

$$
\Theta_{q}(x, t)=2 \int_{0}^{1} \Psi_{q}(x, t, y)\left[\beta(x, t) y^{2}+\delta(x, t)\right] d y .
$$

We put $t=2 a(a>0)$ and $x=r a(r>0)$. Then we get

$$
\left(\frac{a}{2}\right)^{3} \Theta_{q}(r a, 2 a)=\int_{0}^{1}\left(1-y^{2}\right)^{q} \frac{(r+1)^{2}-(6 r+1) y^{2}}{\left[(r+1)^{2}-y^{2}\right]^{5 / 2}} d y .
$$

Applying Lemma 4 with $f(y)=\left(1-y^{2}\right)^{q}, g(y)=(r+1)^{2}-(6 r+1) y^{2}$, and $p(y)=$ $\left[(r+1)^{2}-y^{2}\right]^{-5 / 2}$ yields

$$
\left(\frac{a}{2}\right)^{3} \Theta_{q}(r a, 2 a) \geq \frac{\int_{0}^{1} p(y) f(y) d y \int_{0}^{1} p(y) g(y) d y}{\int_{0}^{1} p(y) d y} .
$$

Since

$\int_{0}^{1} p(y) d y>0, \quad \int_{0}^{1} p(y) f(y) d y>0, \quad$ and $\quad \int_{0}^{1} p(y) g(y) d y=\frac{r^{1 / 2}}{(r+1)^{2}(r+2)^{3 / 2}}>0$,

we conclude that $\Theta_{q}(r a, 2 a)$ is positive. Thus, (3.2)-(3.4) imply that $\left(1 / V_{q}(x)\right)^{\prime \prime}>0$ for $x>0$.

It remains to show that if $-1<q<0$, then $1 / V_{q}$ is not convex on $(0, \infty)$. First, let $-1 / 2<q<0$. We have for $x>0$ :

$$
\int_{0}^{\infty} \frac{e^{-s x} s^{q}}{(x+s)^{3 / 2}} d s \geq \int_{0}^{x} \frac{e^{-s x} s^{q}}{(x+s)^{3 / 2}} d s \geq \int_{0}^{x} \frac{e^{-x^{2}} x^{q}}{(x+s)^{3 / 2}} d s=x^{q-1 / 2} e^{-x^{2}}(2-\sqrt{2}),
$$

so that (2.3) yields $\lim _{x \rightarrow 0}\left(-V_{q}^{\prime}(x)\right)=\infty$. Since $V_{q}(0)=\Gamma(q+1 / 2) / \Gamma(q+1)$, we get

$$
\lim _{x \rightarrow 0}\left(\frac{1}{V_{q}(x)}\right)^{\prime}=\lim _{x \rightarrow 0} \frac{-V_{q}^{\prime}(x)}{\left(V_{q}(x)\right)^{2}}=\infty .
$$

This implies that $\left(1 / V_{q}\right)^{\prime}$ is not increasing on $(0, \infty)$.

Next, let $-1<q \leq-1 / 2$. We assume that $1 / V_{q}$ is convex on $(0, \infty)$. Then we have

$$
\frac{1}{V_{q}((x+y) / 2)} \leq \frac{1}{2}\left(\frac{1}{V_{q}(x)}+\frac{1}{V_{q}(y)}\right) \quad(x, y>0) .
$$

Since $\lim _{y \rightarrow 0} V_{q}(y)=\infty$, we obtain from (3.5):

$$
\frac{1}{V_{q}(x / 2)} \leq \frac{1}{2 V_{q}(x)} \quad(x>0) .
$$

This contradicts Lemma 2.

It is natural also to study properties of $V_{q}(x)$ as function of $q$, where $x>0$ is a fixed number. We now give an affirmative answer to a question posed by Ruskai and Werner [8]: is $V_{q}(x)$ convex with respect to $q$ ? 
THEOREM 3. Let $x>0$ be a real number. The function $q \mapsto V_{q}(x)$ is strictly convex on $(-1, \infty)$.

Proof. Let $x>0$. Since $V_{q}(x)$ is continuous with respect to $q$, it suffices to show that

$$
V_{(a+b) / 2}(x)<\frac{1}{2}\left(V_{a}(x)+V_{b}(x)\right)
$$

for all real numbers $a, b$ with $b>a>-1$. Using (2.2), the integral formula

$$
\frac{1}{x^{r}}=\frac{1}{\Gamma(r)} \int_{0}^{\infty} e^{-x t} t^{r-1} d t \quad(r>0 ; x>0)
$$

and the convolution theorem we get

$$
\begin{aligned}
& \Gamma(a+1) \Gamma(b+1) x^{-(a+b+3 / 2)}\left[V_{a}(x)+V_{b}(x)-2 V_{(a+b) / 2}(x)\right] \\
&= \int_{0}^{\infty} e^{-x s} s^{b} d s \int_{0}^{\infty} e^{-x s} \frac{s^{a}}{(x+s)^{1 / 2}} d s+\int_{0}^{\infty} e^{-x s} s^{a} d s \int_{0}^{\infty} e^{-x s} \frac{s^{b}}{(x+s)^{1 / 2}} d s \\
&-\frac{2 \Gamma(a+1) \Gamma(b+1)}{(\Gamma((a+b) / 2+1))^{2}} \int_{0}^{\infty} e^{-x s} s^{(a+b) / 2} d s \int_{0}^{\infty} e^{-x s} \frac{s^{(a+b) / 2}}{(x+s)^{1 / 2}} d s \\
&= \int_{0}^{\infty} e^{-x t} \sigma_{a, b}(x, t) d t
\end{aligned}
$$

where

$$
\sigma_{a, b}(x, t)=\int_{0}^{t} \frac{1}{(x+s)^{1 / 2}}\left[(t-s)^{b} s^{a}+(t-s)^{a} s^{b}-\frac{2 \Gamma(a+1) \Gamma(b+1)}{(\Gamma((a+b) / 2+1))^{2}}((t-s) s)^{(a+b) / 2}\right] d s .
$$

Let $t>0$. We substitute $s=t(1+y) / 2$ and obtain

$$
\sigma_{a, b}(x, t)=\left(\frac{t}{2}\right)^{a+b+1} \int_{0}^{1} P(x, t, y) Q_{a, b}(y) d y
$$

with

$$
P(x, t, y)=(x+t(1+y) / 2)^{-1 / 2}+(x+t(1-y) / 2)^{-1 / 2}
$$

and

$$
Q_{a, b}(y)=(1-y)^{a}(1+y)^{b}+(1-y)^{b}(1+y)^{a}-\frac{2 \Gamma(a+1) \Gamma(b+1)}{(\Gamma((a+b) / 2+1))^{2}}\left(1-y^{2}\right)^{(a+b) / 2} .
$$

Next, we define for $y \in(0,1)$ :

$$
\begin{aligned}
R_{a, b}(y)= & \left(1-y^{2}\right)^{-(a+b) / 2} Q_{a, b}(y)=\left(\frac{1+y}{1-y}\right)^{(b-a) / 2}+\left(\frac{1-y}{1+y}\right)^{(b-a) / 2} \\
& -\frac{2 \Gamma(a+1) \Gamma(b+1)}{(\Gamma((a+b) / 2+1))^{2}} .
\end{aligned}
$$


Differentiation with respect to $y$ gives

$$
R_{a, b}^{\prime}(y)=\frac{b-a}{1-y^{2}}\left[\left(\frac{1+y}{1-y}\right)^{(b-a) / 2}-\left(\frac{1-y}{1+y}\right)^{(b-a) / 2}\right]>0,
$$

which implies that $y \mapsto R_{a, b}(y)$ is strictly increasing on $(0,1)$. The gamma function is strictly log-convex on $(0, \infty)$, so that we obtain

$$
R_{a, b}(0)=2\left(1-\frac{\Gamma(a+1) \Gamma(b+1)}{(\Gamma((a+b) / 2+1))^{2}}\right)<0 .
$$

Further, we have $\lim _{y \rightarrow 1} R_{a, b}(y)=\infty$. Thus, there exists a number $y_{0} \in(0,1)$ such that $R_{a, b}(y)<0$ for $y \in\left(0, y_{0}\right)$ and $R_{a, b}(y)>0$ for $y \in\left(y_{0}, 1\right)$. Since $y \mapsto P(x, t, y)$ is strictly increasing on $[0,1]$, we get:

$$
\text { if } y \in(0,1), y \neq y_{0}, \quad \text { then } P(x, t, y) Q_{a, b}(y)>P\left(x, t, y_{0}\right) Q_{a, b}(y) .
$$

This leads to

$$
\sigma_{a, b}(x, t)>\left(\frac{t}{2}\right)^{a+b+1} P\left(x, t, y_{0}\right) \int_{0}^{1} Q_{a, b}(y) d y
$$

Using

$$
\begin{gathered}
\int_{0}^{1}\left[(1-y)^{a}(1+y)^{b}+(1-y)^{b}(1+y)^{a}\right] d y=2^{a+b+1} \frac{\Gamma(a+1) \Gamma(b+1)}{\Gamma(a+b+2)}, \\
\int_{0}^{1}\left(1-y^{2}\right)^{(a+b) / 2} d y=\frac{1}{2} \sqrt{\pi} \frac{\Gamma((a+b) / 2+1)}{\Gamma((a+b+1) / 2+1)},
\end{gathered}
$$

and the duplication formula

$$
\Gamma(2 x)=\frac{1}{\sqrt{\pi}} 2^{2 x-1} \Gamma(x) \Gamma(x+1 / 2) \quad(x>0)
$$

we obtain

$$
\int_{0}^{1} Q_{a, b}(y) d y=0 .
$$

From (3.7)-(3.9) we conclude that (3.6) holds.

4. Inequalities and monotonicity. Applying Theorem 2, Lemma 2, and Lemma 5 we are able to extend and to complement inequality (1.2).

THEOREM 4. Let $q \geq 0$ be a real number. Then we have for all $x, y \geq 0$ :

$$
0<\frac{1}{V_{q}(x)}+\frac{1}{V_{q}(y)}-\frac{1}{V_{q}(x+y)} \leq \frac{\Gamma(q+1)}{\Gamma(q+1 / 2)} .
$$

Both bounds are best possible. 
Proof. Let $q, x, y \geq 0$. As remarked in [8], the convexity of $1 / V_{q}$ and the inequality $V_{q}(x / 2)<2 V_{q}(x)$ lead to

$$
\frac{1}{V_{q}(x+y)}<\frac{2}{V_{q}((x+y) / 2)} \leq \frac{1}{V_{q}(x)}+\frac{1}{V_{q}(y)} .
$$

Also, Lemma 5 yields

$$
\frac{1}{V_{q}(x)}+\frac{1}{V_{q}(y)} \leq \frac{1}{V_{q}(x+y)}+\frac{1}{V_{q}(0)}=\frac{1}{V_{q}(x+y)}+\frac{\Gamma(q+1)}{\Gamma(q+1 / 2)} .
$$

Let

$$
w_{q}=V_{q}(x)-\frac{1}{x}
$$

Then we get

$$
\begin{aligned}
& \frac{1}{V_{q}(x)}-\frac{1}{V_{q}(x+y)}=\frac{x(x+y)\left[w_{q}(x+y)-w_{q}(x)\right]-y}{\left[1+x w_{q}(x)\right]\left[1+(x+y) w_{q}(x+y)\right]} \quad \text { and } \\
& \frac{1}{V_{q}(y)}-y=-\frac{y^{2} w_{q}(y)}{1+y w_{q}(y)} .
\end{aligned}
$$

Using (2.6) gives

$$
\lim _{x \rightarrow \infty} x^{3} w_{q}(x)=-\frac{q+1}{2}
$$

This implies

$$
\lim _{y \rightarrow \infty} \lim _{x \rightarrow \infty}\left(\frac{1}{V_{q}(x)}+\frac{1}{V_{q}(y)}-\frac{1}{V_{q}(x+y)}\right)=0 .
$$

If we set $x=y=0$, then equality holds on the right-hand side of (4.1). Thus, the bounds given in (4.1) are sharp.

Since $x \mapsto V_{q}(x)$ is positive and strictly decreasing on $(0, \infty)$, we obtain

$$
V_{q}(x+y)<V_{q}(x)+V_{q}(y) \quad(x, y>0) .
$$

This means that for all $q>-1$ the function $V_{q}$ is strictly subadditive on $(0, \infty)$. However, there is no parameter $q>-1$ such that $V_{q}$ is submultiplicative on $(0, \infty)$. Otherwise, from

$$
V_{q}(x y) \leq V_{q}(x) V_{q}(y) \quad(x, y>0)
$$

we get $V_{q}(1) \leq\left(V_{q}(1)\right)^{2}$ or $1 \leq V_{q}(1)$, which contradicts

$$
V_{q}(x)=\frac{1}{\Gamma(q+1)} \int_{0}^{\infty} e^{-u} \frac{u^{q}}{\left(x^{2}+u\right)^{1 / 2}} d u<\frac{1}{\Gamma(q+1)} \int_{0}^{\infty} e^{-u} \frac{u^{q}}{x} d u=\frac{1}{x} \quad(x>0) .
$$

This leads to the question: do there exist parameters $q$ such that $V_{q}$ is supermultiplicative on $(0, \infty)$ ? The following theorem gives an answer. 
THEOREM 5. Let $q>-1$ be a real number. The function $x \mapsto V_{q}(x)$ is strictly supermultiplicative on $(0, \infty)$, that is,

$$
V_{q}(x) V_{q}(y)<V_{q}(x y) \text { for all } x, y>0
$$

if and only if $q \geq q_{0}$, where $q_{0}=0.72117 \ldots$ is the only solution of $\Gamma(t+1)=\Gamma(t+1 / 2)$ on $(-1 / 2, \infty)$.

Proof. Let $q \geq q_{0}$. We consider two cases. First, let $0<y \leq 1$. Then we obtain

$$
V_{q}(x y) \geq V_{q}(x) \quad \text { and } \quad V_{q}(y)<V_{q}(0)=\frac{\Gamma(q+1 / 2)}{\Gamma(q+1)} .
$$

This implies

$$
V_{q}(x y)-V_{q}(x) V_{q}(y) \geq V_{q}(x)\left(1-V_{q}(y)\right)>V_{q}(x)\left(1-V_{q}(0)\right) .
$$

The function $q \mapsto 1-V_{q}(0)$ is strictly increasing on $(-1 / 2, \infty)$. Hence, we get

$$
1-V_{q}(0) \geq 1-V_{q_{0}}(0)=0 .
$$

Combining (4.5) and (4.6) we obtain $V_{q}(x y)>V_{q}(x) V_{q}(y)$.

Next, let $y>1$. Applying Lemma 2 and (4.3) we get

$$
V_{q}(x y)>V_{q}(x) \frac{1}{y}>V_{q}(x) V_{q}(y) .
$$

It remains to show: if (4.4) holds, then $q \geq q_{0}$. Again, we consider two cases. Let $q>-1 / 2$. We set $x=y$ in (4.4) and let $x$ tend to 0 . This leads to $V_{q}(0) \leq 1=V_{q_{0}}(0)$. Thus, $q \geq q_{0}$.

Now, we assume that $-1<q \leq-1 / 2$. We prove that the inequality

$$
V_{q}(x / 2)<V_{q}(1 / 2) V_{q}(x)
$$

is valid for all sufficiently small $x$. Using (1.1) we conclude that (4.7) is equivalent to

$$
0<\int_{x}^{\infty}\left(t^{2}-x^{2}\right)^{q}\left[V_{q}(1 / 2) e^{3 x^{2} / 4-t^{2}}-2^{-2 q-1} e^{-t^{2} / 4}\right] d t=I_{q}(x), \quad \text { say } .
$$

We define

$$
z(q)=-\frac{\log \left(4^{q} V_{q}(1 / 2)\right)}{\log (4)}
$$

From (2.5) we obtain

$$
2 \Gamma(q+1)\left[4^{q} V_{q}(1 / 2)-1 / 2\right]=\int_{0}^{\infty} e^{-s / 4} \frac{s^{q}}{(1+s)^{1 / 2}} d s-\int_{0}^{\infty} e^{-s} s^{q} d s .
$$

Since $e^{-s / 4} /(1+s)^{1 / 2}>e^{-s}$ for $s>0$, we get

$$
4^{q} V_{q}(1 / 2)>1 / 2 \text { for } q>-1 \text {. }
$$


This implies $z(q)<1 / 2$. Let $\omega=\omega(q)$ be a real number such that

$$
z(q)<\omega<1 / 2
$$

We have

$$
I_{q}(x)=\int_{x}^{\infty} A_{q}(x, t) B_{q}(x, t) d t
$$

where

$$
A_{q}(x, t)=\left(t^{2}-x^{2}\right)^{q+\omega} \quad \text { and } \quad B_{q}(x, t)=\left(t^{2}-x^{2}\right)^{-\omega}\left[V_{q}(1 / 2) e^{3 x^{2} / 4-t^{2}}-2^{-2 q-1} e^{-t^{2} / 4}\right] .
$$

Since $q+\omega \leq-1 / 2+\omega<0$, we conclude that $t \mapsto A_{q}(x, t)$ is strictly decreasing on $(x, \infty)$. Moreover, the function

$$
t \mapsto b_{q}(x, t)=\left(t^{2}-x^{2}\right)^{\omega} e^{t^{2}} B_{q}(x, t)
$$

is strictly decreasing on $[x, \infty)$ with $\lim _{t \rightarrow \infty} b_{q}(x, t)=-\infty$. Applying (4.8) yields $b_{q}(x, x)>0$. Thus, there exists a number $t_{0}>x$ such that $b_{q}(x, t)$ is positive for $t \in\left(x, t_{0}\right)$ and negative for $t \in\left(t_{0}, \infty\right)$. This implies

$$
A_{q}(x, t) B_{q}(x, t)>A_{q}\left(x, t_{0}\right) B_{q}(x, t) \text { for } \quad x<t \neq t_{0} .
$$

Hence, we obtain

$$
I_{q}(x)>A_{q}\left(x, t_{0}\right) \int_{x}^{\infty}\left(t^{2}-x^{2}\right)^{-\omega}\left[V_{q}(1 / 2) e^{3 x^{2} / 4-t^{2}}-2^{-2 q-1} e^{-t^{2} / 4}\right] d t=A_{q}\left(x, t_{0}\right) J_{q}(x),
$$

say.

We have

$$
2 J_{q}(0)=\Gamma(1 / 2-\omega)\left[V_{q}(1 / 2)-4^{-(q+\omega)}\right] .
$$

Since $V_{q}(1 / 2)>4^{-(q+\omega)}$ is equivalent to $\omega>z(q)$, we conclude from (4.9) and (4.11) that $J_{q}(0)>0$. This implies that there is a number $\epsilon>0$ such that $J_{q}(x)$ is positive for $x \in(0, \epsilon)$. From (4.10) we get $I_{q}(x)>0$ for $x \in(0, \epsilon)$. The proof of Theorem 5 is complete.

REMARK 3. Comments on the relevance of sub- and supermultiplicative functions in various fields as well as references on this subject can be found in [6].

REMARK 4. Inequality (4.2) can be improved. In fact, from

$$
\frac{2}{\left((x+y)^{2}+u\right)^{1 / 2}}<\frac{1}{\left(x^{2}+u\right)^{1 / 2}}+\frac{1}{\left(y^{2}+u\right)^{1 / 2}} \quad(x, y, u>0)
$$

and (2.5) we obtain for all $q>-1$ :

$$
2<\frac{V_{q}(x)+V_{q}(y)}{V_{q}(x+y)} \quad(x, y>0) .
$$


Let $q>-1 / 2$ and $x=y$. If we let $x$ tend to 0 , then the ratio on the right-hand side of (4.12) converges to 2 . Thus, (at least) for $q>-1 / 2$ the lower bound 2 cannot be replaced by a larger term, which is independent of $x$ and $y$.

We now present an inequality which reveals a connection between $\left(V_{q}^{(k)}(x)\right)^{n}$ and $\left(V_{q}^{(n)}(x)\right)^{k}$.

THEOREM 6. The inequality

$$
(-1)^{n k}\left(\frac{V_{q}^{(k)}(x)}{V_{q}(x)}\right)^{n} \leq(-1)^{n k}\left(\frac{V_{q}^{(n)}(x)}{V_{q}(x)}\right)^{k}
$$

holds for all real numbers $x>0$ and integers $n, k$ with $n \geq k \geq 0$ if and only if $q \in(-1,0]$.

Proof. Let $q \in(-1,0], x>0$, and $n \geq k \geq 0$. Applying Theorem 1 and Lemma 6 we conclude that (4.13) is valid. Conversely, we assume that (4.13) holds for all $x>0$ and $n, k$ with $n \geq k \geq 0$. We set $n=2$ and $k=1$ and obtain

$$
\left(V_{q}^{\prime}(x)\right)^{2} \leq V_{q}(x) V_{q}^{\prime \prime}(x)
$$

This means that $V_{q}$ is log-convex on $(0, \infty)$, so that Remark 2 implies $q \in(-1,0]$.

Finally, we study the monotonicity behaviour of the ratio $V_{p} / V_{q}$ and the difference $V_{p}-V_{q}$.

THEOREM 7. Let $p, q>-1$ be real numbers.

(i) The function $x \mapsto V_{p}(x) / V_{q}(x)$ is increasing on $(0, \infty)$ if and only if $p \geq q$.

(ii) The function $x \mapsto V_{p}(x)-V_{q}(x)$ is increasing on $(0, \infty)$ if and only if $p \geq q$. If $p>q>-1$, then $V_{p} / V_{q}$ and $V_{p}-V_{q}$ are strictly increasing on $(0, \infty)$.

Proof. Since the proofs of (i) and (ii) are similar, we only establish part (i). First, we assume that $p>q>-1$. Applying (2.2), (2.3), and the convolution theorem we get for $x>0$ :

$$
\begin{aligned}
\Gamma(p+1) & \Gamma(q+1) x^{-(p+q+1)}\left(V_{q}(x)\right)^{2}\left(\frac{V_{p}(x)}{V_{q}(x)}\right)^{\prime}=V_{p}^{\prime}(x) V_{q}(x)-V_{p}(x) V_{q}^{\prime}(x) \\
= & \int_{0}^{\infty} e^{-x s} \frac{s^{p}}{(x+s)^{1 / 2}} d s \int_{0}^{\infty} e^{-x s} \frac{s^{q}}{(x+s)^{3 / 2}} d s \\
& -\int_{0}^{\infty} e^{-x s} \frac{s^{q}}{(x+s)^{1 / 2}} d s \int_{0}^{\infty} e^{-x s} \frac{s^{p}}{(x+s)^{3 / 2}} d s=\int_{0}^{\infty} e^{-x t} \Delta_{p, q}(x, t) d t,
\end{aligned}
$$

where

$$
\Delta_{p, q}(x, t)=\int_{0}^{t} \frac{s^{q}(t-s)^{q}}{(x+s)^{3 / 2}(x+t-s)^{1 / 2}}\left[(t-s)^{p-q}-s^{p-q}\right] d s .
$$

The substitution $s=t(1+y) / 2$ leads to

$$
\Delta_{p, q}(x, t)=\left(\frac{t}{2}\right)^{p+q+1} \int_{-1}^{1} \phi_{q}(x, t, y)(x+t(1-y) / 2)\left[(1-y)^{p-q}-(1+y)^{p-q}\right] d y
$$


with

$$
\phi_{q}(x, t, y)=\frac{\left(1-y^{2}\right)^{q}}{[(x+t(1-y) / 2)(x+t(1+y) / 2)]^{3 / 2}} .
$$

Since $y \mapsto \phi_{q}(x, t, y)$ is even and $y \mapsto(1-y)^{p-q}-(1+y)^{p-q}$ is odd, we obtain

$$
\Delta_{p, q}(x, t)=-2\left(\frac{t}{2}\right)^{p+q+2} \int_{0}^{1} \phi_{q}(x, t, y) y\left[(1-y)^{p-q}-(1+y)^{p-q}\right] d y>0 .
$$

From (4.14) and (4.15) we conclude that $\left(V_{p}(x) / V_{q}(x)\right)^{\prime}>0$ for $x>0$.

We define

$$
h_{q}(x)=V_{q}(x)-\frac{1}{x}+\frac{q+1}{2 x^{3}} .
$$

Then, (2.6) gives

$$
h_{q}(x)=O\left(\frac{1}{x^{5}}\right)
$$

If $x \mapsto V_{p}(x) / V_{q}(x)$ is increasing on $(0, \infty)$, then we get for all $x>0$ :

$$
\begin{aligned}
0 \leq & {\left[V_{p}(2 x) V_{q}(x)-V_{p}(x) V_{q}(2 x)\right] x^{4} } \\
= & {\left[h_{p}(2 x) h_{q}(x)-h_{p}(x) h_{q}(2 x)\right] x^{4}+\left[-h_{p}(x) / 2+h_{p}(2 x)+h_{q}(x) / 2-h_{q}(2 x)\right] x^{3} } \\
& +\left[(q+1) h_{p}(x) / 8-(q+1) h_{p}(2 x)-(p+1) h_{q}(x) / 8\right. \\
& \left.+(p+1) h_{q}(2 x)\right] x / 2+3(p-q) / 16 .
\end{aligned}
$$

Applying (4.16) we obtain that the expression on the right-hand side converges to $3(p-q) / 16$, if $x$ tends to $\infty$. Thus, $p \geq q$.

Acknowledgements. I am grateful to Professor M. B. Ruskai for providing the short and elegant proof that for $q \in(-1,0)$ the function $1 / V_{q}$ is not convex on $(0, \infty)$. Also, I thank the referee for helpful comments.

\section{REFERENCES}

1. H. Alzer and C. Berg, Some classes of completely monotonic functions, Ann. Acad. Scient. Fennicae 27 (2002), 445-460.

2. T. Bang and B. Fuglede, No two quotients of normalized binomial mid-coefficients are equal, J. Number Th. 35 (1990), 345-349.

3. R. Brummelhuis and M. B. Ruskai, A simple one-dimensional model for atoms in strong magnetic fields, Contemporary Math. 217 (1998), 109-119.

4. R. Brummelhuis, M. B. Ruskai and E. Werner, One dimensional regularizations of the Coulomb potential with application to atoms in strong magnetic fields, Studies Adv. Math. 16 (2000), 67-75.

5. A. M. Fink, Kolmogorov-Landau inequalities for monotone functions, J. Math. Anal. Appl. 90 (1982), 251-258.

6. C. E. Finol and M. Wójtowicz, Multiplicative properties of real functions with applications to classical functions, Aequat. Math. 59 (2000), 134-149. 
7. D. S. Mitrinović, Analytic inequalities (Springer-Verlag, 1970).

8. M. B. Ruskai and E. Werner, Study of a class of regularizations of $1 /|x|$ using Gaussian integrals, SIAM J. Math. Anal. 32 (2000), 435-463.

9. D. V. Widder, The Laplace transform (Princeton Univ. Press, Princeton, 1941).

10. M. Wirth, On considère la fonction de $\mathbf{R}$ dans $\mathbf{R}$ défine par $f(x)=e^{-x^{2} / 2}$; démontrer que la fonction $g$ de $\mathbf{R}$ dans $\mathbf{R}$ défine par $g(x)=f(x) / \int_{x}^{\infty} f(t) d t$ est convexe, Rev. Math. Spéciales 104 (1993), 187-188. 